\title{
O MÉTODO PILATES NO SOLO NA QUALIDADE DE VIDA, FUNÇÃO PULMONAR E FORÇA MUSCULAR RESPIRATÓRIA DE IDOSAS
}

\section{Mariana Barcelos Fabrício dos Santos}

Centro Universitário de Maringá - UniCesumar, Maringá - PR, Brasil.

\section{Mateus Dias Antunes}

Universidade de São Paulo - USP, Brasil.

\section{Daniel Vicentini de Oliveira}

Centro Universitário Metropolitano de Maringá UNIFAMMA. Pós-doutorando em Promoção da Saúde pelo Centro Universitário de Maringá - UniCesumar, Maringá - PR, Brasil

\section{Siméia Gaspar Palácio}

Centro Universitário de Maringá - UniCesumar, Maringá - PR, Brasil.
RESUMO: Este estudo teve o objetivo de analisar os efeitos do método Pilates no solo na qualidade de vida, função pulmonar e força muscular respiratória em mulheres idosas. Estudo quasi-experimental, realizado com 19 idosas no município de Maringá, Paraná, avaliadas por meio do SF-36, da espirometria e manovacuometria. As idosas foram submetidas a dez sessões de Pilates no solo, realizadas três vezes por semana, durante 60 minutos. Ao término do tratamento as pacientes foram reavaliadas e os dados analisados pelos testes de Wilcoxon e Mann Whitney $(\mathrm{p}<0,05)$. Houve melhora da força muscular inspiratória $(40,5 \%)$, expiratória $(42,9 \%)$, da capacidade vital forçada $(9,1 \%)$, do volume expiratório forçado no primeiro minuto $(13,3 \%)$; e principalmente na qualidade de vida nos domínios de limitação por aspectos físicos $(68,42 \%)$, aspectos emocionais ( $43,42 \%)$, capacidade funcional $(35,0 \%)$ e dor $(34,37 \%)$. O método Pilates no solo mostrouse eficaz para melhorar a qualidade de vida, na função pulmonar e força muscular respiratória.

PALAVRAS-CHAVE: Envelhecimento; Atividade motora; Exercício.

\section{PILATES METHOD IN LIFE QUALITY, LUNG FUNCTION AND RESPIRATORY MUSCULAR FORCE IN ELDERLY PEOPLE}

\begin{abstract}
The effects of the Pilates method on life quality, lung functions and respiratory muscular force in elderly females were analyzed. The quasi-experimental study was undertaken with 19 elderly women in Maringá, Brazil, and evaluated by SF-36, spirometry and manovacuometry. Elderly females underwent ten Pilates sessions on the ground, during $60 \mathrm{~min}$, three times a week. Patients were then re-evaluated and data analyzed by Wilcoxon's and Mann Whitney's tests $(\mathrm{p}<0.05)$. Improvement in inspiratory muscular force $(40.5 \%)$, expiratory (42.9\%) muscular force, forced vital ability (9.1\%), forced expiratory volume at the first minute (13.3\%) and mainly in life quality in domains limitation by physical aspects $(68.42 \%)$, emotional aspects (43.42\%), functional ability (35.0\%) and pain (34.37\%). Pilates method was efficient to improve life quality, and for lung functioning and respiratory muscular force.
\end{abstract}

KEY WORDS: Aging; Motor activity; Exercise.

\section{INTRODUÇÃO}

Autor correspondente:

Daniel Vicentini de Oliveira

d.vicentini@hotmail.com
O envelhecimento biológico humano é dinâmico e progressivo, sendo caracterizado por involuções morfológicas e funcionais no organismo que afetam a maioria dos órgãos, as quais levam a um 
declínio acentuado das adaptações homeostáticas ${ }^{1-2}$. Algumas dessas alterações presentes no envelhecimento ocorrem principalmente no sistema respiratório, pois, a capacidade pulmonar sofre redução gradativa com a idade, visto que, ao chegar aos 70 anos, os pulmóes operam com apenas um terço da capacidade de expansão de quando eram mais novos ${ }^{3}$.

As alterações mais importantes que ocorrem no sistema respiratório são a diminuição da retração elástica dos pulmões, a redução da complacência da caixa torácica e declínio da força muscular ventilatória. O tamanho máximo dos pulmões, ou a capacidade pulmonar total não muda com a idade, mas a capacidade residual funcional e o volume residual aumentam, enquanto, a capacidade inspiratória e a capacidade vital diminuem ${ }^{4}$.

$\mathrm{O}$ aumento na rigidez da caixa torácica e a diminuição significativa da força dos músculos respiratórios levam a uma redução progressiva da função pulmonar nos indivíduos idosos. Entre as principais alterações observadas pode-se citar: a redução progressiva da capacidade vital e da capacidade inspiratória; o aumento do volume residual; a redução do volume expiratório forçado no primeiro segundo (VEF1) e o fluxo expiratório forçado de $25 \%$ a $75 \%$ (FEF 25-75) da manobra de capacidade vital forçada (CVF) ${ }^{5}$.

A perda funcional respiratória decorrente dessas complicações são fatores que influenciam diretamente na qualidade de vida do idoso ${ }^{6,8-9}$. Dessa forma, a realização de exercícios físicos ajuda na proteção contra o declínio cronológico dos sistemas orgânicos e no retardo da perda funcional da população idosa ${ }^{10}$.

Dentre as alternativas terapêuticas disponíveis para o tratamento dos idosos, cita-se o método Pilates que busca o condicionamento físico e mental, e possui como intuito principal ampliar a independência no cotidiano das atividades de vida diária ${ }^{11}$. Esse método baseia-se no fortalecimento do centro de força, expressão que denota a circunferência do tronco inferior, estrutura de suporte para o corpo. Por meio desse método, há a melhora da capacidade pulmonar, pelo fortalecimento dos músculos respiratórios, da mobilidade, pois exercita a tonificação muscular e, da flexibilidade, por meio de equipamentos diferenciados, como a bola de Pilates ${ }^{12-13}$.

Porém, são extremamente escassos estudos que relatam efeitos do método Pilates, principalmente no solo, e com idosas; além disso, são poucos também os estudos nesta temática, com variáveis relacionadas à função e força muscular, o que justifica a realização desta pesquisa. Dessa forma, de acordo com os benefícios terapêuticos relatados pela literatura com a prática de atividade física em pessoas idosas, este estudo teve como objetivo analisar os efeitos do método Pilates no solo na qualidade de vida, função pulmonar e força muscular respiratória em mulheres idosas.

\section{METODOLOGIA}

\section{PARTICIPANTES}

Foram selecionadas por conveniência 19 mulheres com idade de 60 a 75 anos do município de Maringá, Paraná, triadas da clínica escola de Fisioterapia do Centro Universitário de Maringá (UniCesumar), e da comunidade em geral, mediante a divulgação do estudo por meio de panfletos informativos. Foram excluídas do estudo mulheres com deficiência física, debilidade cognitiva e com diabetes e ou hipertensão arterial descompensada.

\section{INSTRUMENTOS}

Após apresentar os objetivos da pesquisa, as idosas, que aceitaram participar da mesma, assinaram o Termo de Consentimento Livre e Esclarecido (TCLE). As pacientes idosas foram submetidas a uma avaliação da capacidade pulmonar por meio da manovacuometria e da espirometria.

Para a avaliação da qualidade de vida foi aplicado o questionário SF-36, composto por oito domínios, sendo estes: capacidade funcional; aspectos físicos; dor; estado geral da saúde; vitalidade; aspectos sociais; aspectos emocionais e saúde mental. A pontuação varia de 0 a 100, sendo que 0 corresponde ao pior estado de saúde e 100 o melhor ${ }^{14}$.

Em seguida, para avaliar a capacidade pulmonar foram realizadas a manovacuometria ${ }^{15}$ e a espirometria ${ }^{16}$. Através do manovacuômetro mensurou-se a força da 
musculatura inspiratória e expiratória, determinada pela pressão negativa e pressão positiva. Para a verificação da força muscular da pressão inspiratória (PImáx), a participante foi posicionada sentada, com o tronco em um ângulo de $90^{\circ}$ graus com as coxas, braços relaxados na lateral do tronco e com o nariz ocluído por um clipe nasal. A mesma foi então instruída a expirar até alcançar o volume residual, enquanto na verificação da pressão expiratória máxima (PEmáx), a avaliada ainda sentada, realizou inspiração até alcançar a capacidade pulmonar total e, em seguida, realizou uma expiração máxima. Para a avaliação da pressão inspiratória máxima (PImáx) e da PEmáx, solicitou-se a paciente que realizasse o teste por três vezes consecutivas, considerando como resposta a média dos três valores. Os valores normais padrões da força dos músculos inspiratórios, expressos na PImáx variam entre $-90 \mathrm{cmH} 20$ a $-120 \mathrm{cmH} 2 \mathrm{O}$ e para PEmáx entre $+100 \mathrm{a}+150 \mathrm{cmH} 2 \mathrm{O}$. Sendo assim, quanto maior o valor obtido no exame, melhor a força desses músculos ${ }^{15}$.

Para a realização da espirometria, foi utilizado o espirômetro da marca Micro Medical ${ }^{\circledR}$ em que a paciente foi instruída a permanecer sentada, com o tronco em $90^{\circ}$ graus com as coxas, braço esquerdo relaxado e braço direito segurando o aparelho, ocluindo o nariz por um clipe nasal. Logo depois, solicitou-se uma respiração oral em volume corrente por três ciclos respiratórios, seguido de uma inspiração profunda até a capacidade pulmonar total e, na sequência, o paciente realizou uma expiração forçada, por cerca de 06 segundos, até o volume residual, com a boca acoplada no bocal do espirômetro. O teste foi repetido três vezes, sendo considerado o maior valor obtido para a variável CVF. Durante a avaliação houve incentivo verbal por parte do avaliador para que fosse obtido o maior esforço possível ${ }^{16}$.

Para avaliar a capacidade pulmonar foram consideradas três variáveis no teste: CVF, VEF1 e FER (Índice de Tiffeneau). As três variáveis foram graduadas em porcentagem em relação a um valor previsto para cada paciente considerando a estatura e a idade específicas. O valor de CVF foi usado para graduar a presença de distúrbios respiratórios de restrição. Os valores de graduação variaram de normal: $(\geq 80 \%)$, restrição leve (80-60\%); restrição moderada (60-40\%) e grave $(\leq 40)$. O valor de VEF1 foi usado para graduar um distúrbio respiratório obstrutivo, sendo que os valores de graduação variam de normal $(\geq 80 \%)$, obstrução leve $(80-60 \%)$; obstrução moderada $(60-50 \%)$ e grave $(\leq 50)$. Quanto maior a porcentagem dos valores de CVF e VEF1 melhor classificou-se a capacidade pulmonar do paciente. A relação entre esses dois parâmetros indicou o FER que foi graduado conforme a idade e a altura do paciente, sendo que o referencial de normalidade adotado variou de $68 \%$ a $85 \%$ de CVF e para verificar a porcentagem de melhora dos resultados obtidos consideraram-se os valores de pré e pós-intervenção ${ }^{16}$.

No que diz respeito às variáveis espirométricas: CVF, VEF1 e FER das 19 voluntárias idosas, realizou-se a graduação dos dados em relação a valores previstos específicos para cada paciente, considerando a altura e a idade. Dessa forma, as respostas ventilatórias no pré e pós-intervenção do exercício físico variaram conforme as alterações anatômicas do esqueleto torácico de cada paciente. Sendo assim, a rigidez aumentada do tórax e a complacência decrescente da parede torácica variaram individualmente ${ }^{16}$.

\section{PROCEDIMENTOS}

Este estudo de intervenção foi realizado na Clínica Escola de Fisioterapia do Centro Universitário de Maringá (UNICESUMAR), após a aprovação do Comitê de Ética da mesma instituição de ensino sob parecer de número 1.359.841/2015.

Ao término da avaliação, iniciaram-se as dez sessões com o método Pilates no solo durante três vezes semanais com duração de 60 minutos. Ao término do período das sessões, as pacientes foram reavaliadas pelos mesmos instrumentos utilizados na avaliação inicial para verificação dos resultados alcançados. A avaliação foi feita um dia após o último dia de intervenção.

O programa de exercícios partiu de um protocolo pré-estabelecido com progressão individualizada de carga e do número de séries e repetições a cada três sessões. O roteiro de exercícios incluiu uma sequência de relaxamento inicial, alongamento e fortalecimento de assoalho pélvico e dos membros inferiores, seguido de fortalecimento de tronco alternando as posições: deitado 
em decúbito dorsal; sentado na bola suíça e fortalecimento da musculatura dorsal em decúbito ventral, terminando com relaxamento.

Os alongamentos incluíram a musculatura cervical, de tronco, de cadeia posterior da coluna e dos membros inferiores. Os exercícios do método Pilates inclusos no protocolo foram: fole; heel tap com a bola suíça, ponte, rolamento lateral; ondulação do corpo com a bola suíça e ondulação completa. Além destes, também foram realizados: abdominal fabuloso na bola suíça; abdominal no solo com magic circle; série single leg drop; série side leg; golfinho; estrela e exercícios sentados na bola para equilíbrio dinâmico.

\section{ANÁLISE DE DADOS}

Todas as análises foram realizadas no software SPSS, versão 22.0. Inicialmente, foi verificada a normalidade dos dados por meio do teste de ShapiroWilk. Como os dados apresentaram distribuição normal, foi utilizado o teste $\mathrm{t}$ de student dependente para comparar a diferença relativa entre as médias pré e pósintervenção, obtendo resultados em porcentagem, e interpretando-os como melhora dos pacientes tratados, adotando como parâmetro o nível de significância de 5\%.

\section{RESULTADOS}

Os resultados obtidos no presente estudo puderam confirmar a eficácia da aplicação do método Pilates no solo em mulheres idosas saudáveis, pois houve melhora estatisticamente significante da PImáx $(\mathrm{p}<0,001)$ e da PEmáx $(\mathrm{p}<0,001)$ por meio da mensuração da manovacuometria. Nesse teste, a diferença relativa das médias representou uma melhora de 40,5\% $\pm 31,7 \mathrm{cmH} 20$ para PImáx, e de 42,9\% $\pm 37,0 \mathrm{cmH} 20$ para PEmáx, conforme ilustrado na Tabela 1.

\section{TABELA 1}

Em relação aos parâmetros ventilatórios analisados pela espirometria, houve aumento dos valores de: CVF; VEF1 e FER ao comparar a média dos resultados pré e pós-intervenção, sendo então 9,1\%; $13,3 \%$ e de $3,6 \%$, respectivamente. No entanto, observouse melhora estatisticamente significante apenas no volume expiratório forçado no primeiro minuto (VEF1), conforme observado na Tabela $2(\mathrm{p}<0,001)$.

A maioria das pacientes $(94,7 \%)$ não apresentou distúrbios ventilatórios obstrutivos ou restritivos tanto na pré como na pós-intervenção.

Tabela 1. Média das variáveis PImáx e PEmáx no pré e pósintervenção

\begin{tabular}{|c|c|c|c|c|c|}
\hline 莺 & 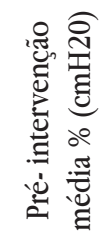 & 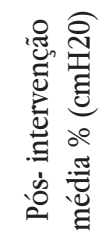 & 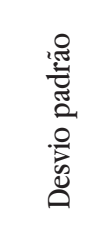 & 䓂 & 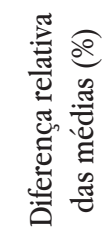 \\
\hline PImáx & 50,1 & 66,3 & $(31,7)$ & $<0,001$ & 40,5 \\
\hline PEmáx & 63,6 & 89,6 & $(37,0)$ & $<0,001$ & 42,9 \\
\hline
\end{tabular}

Em relação à qualidade de vida (Tabela 3), verificou-se diferença significativa $(\mathrm{p}<0,05)$ em todos os domínios do questionário SF-36 do pró para o pós-teste, indicando aumento nos escores após a intervenção. Vale ressaltar que os maiores percentuais de variação foram nos domínios de limitação por aspectos físicos $(68,42 \%)$, aspectos emocionais $(43,42 \%)$, capacidade funcional $(35,0 \%)$ e dor $(34,37 \%)$. 
Tabela 2. Média das variáveis espirométricas no pré e pósintervenção

PVF
CVF

Tabela 3. Média dos domínios do Questionário SF- 36 no pré e pós-intervenção

\begin{tabular}{|c|c|c|c|c|c|}
\hline 总 & 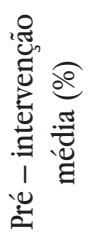 & 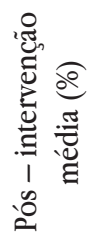 & 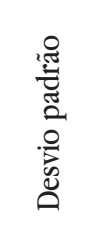 & 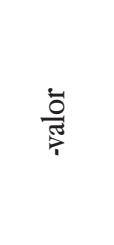 & 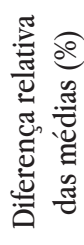 \\
\hline $\begin{array}{l}\text { Capacidade } \\
\text { funcional }\end{array}$ & 58,4 & 93,4 & $(23,5)$ & $<0,001$ & $\begin{array}{c}35,0 \\
\%\end{array}$ \\
\hline $\begin{array}{l}\text { Aspectos } \\
\text { físicos }\end{array}$ & 26,3 & 94,7 & $(36,2)$ & $<0,001$ & $\begin{array}{c}68,4 \\
\%\end{array}$ \\
\hline Dor & 40,2 & 74,5 & $(18,3)$ & $<0,001$ & $\begin{array}{c}34,4 \\
\%\end{array}$ \\
\hline $\begin{array}{l}\text { Estado geral } \\
\text { de saúde }\end{array}$ & 61,7 & 82,3 & $(21,6)$ & 0,001 & $\begin{array}{c}20,6 \\
\%\end{array}$ \\
\hline Vitalidade & 49,2 & 81,58 & $(17,6)$ & $<0,001$ & $\begin{array}{c}32,4 \\
\%\end{array}$ \\
\hline $\begin{array}{l}\text { Aspectos } \\
\text { sociais }\end{array}$ & 61,8 & 93,0 & $(22,8)$ & $<0,001$ & $\begin{array}{c}31,2 \\
\%\end{array}$ \\
\hline $\begin{array}{l}\text { Aspectos } \\
\text { emocionais }\end{array}$ & 56,6 & 100,0 & $(46,0)$ & 0,001 & $\begin{array}{c}43,4 \\
\%\end{array}$ \\
\hline $\begin{array}{l}\text { Saúde } \\
\text { mental }\end{array}$ & 56,6 & 77,9 & $(23,9)$ & 0,001 & $\begin{array}{c}21,3 \\
\%\end{array}$ \\
\hline
\end{tabular}

*Diferença estatisticamente significativa pelo teste t/Mann-Whitnney.

\section{DISCUSSÃO}

No presente estudo observou-se a efetividade da intervenção do método Pilates no solo sobre os parâmetros respiratórios de mulheres idosas saudáveis, uma vez que houve aumento da força muscular tanto inspiratória como expiratória, além da melhora da capacidade pulmonar dessas pacientes. Os resultados obtidos na porcentagem de melhora da força muscular respiratória foram de 40,46\% cmH20 na PImáx e de 42,93\% cmH20 na PEmáx. A justificativa desse progresso pode ser atribuída a um dos princípios norteadores desse método: a técnica respiratória denominada de "respiração lateral", associada à expiração máxima durante os exercícios ${ }^{16}$.

A respiração é o fator primordial no início do movimento, pois fornece a organização do tronco pelo recrutamento dos músculos estabilizadores profundos da coluna e da caixa torácica, favorecendo a expansão pulmonar ${ }^{17}$. pela contração ativa desses músculos, em um estudo $^{16}$, autores observaram que a técnica respiratória do método Pilates permite maior expansão torácica na inspiração e na expiração maior retração torácica, o que promove maior mobilidade toracoabdominal durante os exercícios. O aumento da Pimáx também é justificado pelo recrutamento do diafragma, que permanece na posição de alongamento durante a realização dos exercícios do Pilates solo em retroversão pélvica ${ }^{19-20}$. Além disso, a contração da musculatura abdominal promove o fortalecimento do diafragma, por meio do aumento da pressão intraabdominal o que, consequentemente, aprimora a capacidade pulmonar inspiratória ${ }^{21-22}$.

Os achados desta investigação evidenciaram um leve aumento para os parâmetros espirométricos sendo estes: 9,07\% para CVF, 13,25\% para VEF1 e 3,62\% para FER. No presente estudo, a manutenção de valores baixos para FER está relacionada ao fato desse índice depender da divisão das duas grandezas: VEF1/CVF. Sendo assim, a porcentagem de melhora do FER é diretamente proporcional ao valor de VEF1 e inversamente proporcional à CVF. Como ambos os valores de VEF1 e CVF tiveram progresso quase equivalente na porcentagem, a variação pré e pós-intervenção de FER foi a menor dentre todas as variáveis estudadas. Logo, o método Pilates promove reeducação e coordenação da 
mecânica respiratória aprimorando assim a ventilação pulmonar e, consequentemente, a nutrição tecidual dos músculos do corpo ${ }^{24}$.

Quanto à qualidade de vida os resultados do questionário SF- 36 foram similares aos encontrados no estudo de Nascimento e Carvalho ${ }^{25}$, no qual os, autores ao compararem um grupo ativo de 19 integrantes idosas com idade entre 60-69 anos com outro iniciante de 21 idosas, antes e após a participação de um programa de exercícios fundamentado no método Pilates, verificaram a evolução percentual nos seguintes domínios: capacidade funcional de 8,4\%; aspectos físicos de 17,1\%; dor de 15,5\% e no estado geral de saúde de 32,6\%. Uma explicação para esses achados é que a prática do exercício físico, em idade avançada, traz benefícios psicossociais, reduzindo assim os níveis de ansiedade e estresse do indivíduo ${ }^{26}$.

Nesta mesma linha de pensamento, ao compararem em um estudo ${ }^{27}$ a percepção da qualidade de vida entre idosos sedentários e idosos praticantes regulares de exercício físico, observaram que a prática de atividade física intervém positivamente na forma como a pessoa percebe sua vida diária, influenciando, por conseguinte, na adoção de um novo estilo de vida.

Os autores reafirmam os efeitos benéficos do exercício físico pelo método Pilates ao estudarem um grupo de mulheres sedentárias e ativas fisicamente, obtendo melhores índices de percepção da qualidade de vida nas que eram fisicamente ativas ${ }^{28}$. A análise estatística evidenciou que as idosas praticantes do Pilates obtiveram a média de 100 pontos no momento da reavaliação para as dimensões aspectos físicos e aspectos emocionais.

Como implicações práticas deste estudo, destaca-se a possibilidade de um fisioterapeuta basear no protocolo desta intervenção e aplicá-lo em sua prática clínica, podendo também aprimorar, para melhorar a saúde física e mental dos idosos. A participação de idosos em grupos de convivência não se limita à promoção da saúde, uma vez que esses locais significam para os idosos tempo e espaço à escuta e o estabelecimento de novas amizades, representando incremento na sua qualidade de vida ${ }^{27}$. Pelo reduzido tamanho amostral, sugere-se que novos estudos sejam realizados com o mesmo enfoque terapêutico abordado. Além disso, a ausência de um grupo controle pode ser apontada como uma limitação do estudo, assim como o tamanho amostral reduzido, com uma amostra não probabilística.

\section{CONCLUSÃO}

Diante do exposto, pode-se concluir que o método Pilates contribuiu significativamente para a melhora da capacidade pulmonar, da força muscular respiratória e da qualidade de vida das pacientes tratadas.

\section{REFERÊNCIAS}

1. Moraes EM. Fisiologia do Envelhecimento aplicada à prática clínica. In: Silva ALA. Princípios Básicos de Geriatria e Gerontologia. Belo Horizonte: Coopmed; 2008.Cap.4.p.37-62.

2. Netto MP, Filho ETC. Geriatria: Fundamentos, Clínica e Terapêutica. São Paulo: Atheneu, 2005.

3. Marques D. Análise da capacidade funcional de mulheres idosas praticantes do método Pilates. [citado 2013 Mar 18]. Disponível em: //http:// repositorio.unesc.net/handle/1/1474. (acesso em 18 março 2017).

4. Fagherazzi SB. Análise da Influência de diferentes fatores sobre as pressões ventilatórias máximas em idosos do município de Porto Alegre-Brasil [dissertação]. Porto Alegre: Pontifícia Universidade Católica do Rio Grande do Sul; 2010.

5. Ruivo S, Viana P, Martins C, Baeta C. Effects of aging on lung function. A comparison of lung function in healthy adults and the elderly. Rev Port Pneum. 2009;15(4):629-53.

6. Spirduzo WW. Dimensões físicas do envelhecimento. $2^{\mathrm{a}}$ ed. Barueri: Manole, 2005.

7. Eurico STL, Souza CCT, Silva VLR. Efeitos de 20 sessões do método Pilates no alinhamento postural e flexibilidade de mulheres jovens: estudo piloto. Fisioter Pesq. 2013;20(2):143-50.

8. Zalli M, Benin AC, Gomes GD, Matos FM. Geriatria para Clínicos: medicina aplicada à Terceira Idade. $2^{\mathrm{a}}$ ed. Santa Catarina: Revinter; 2012. 
9. Curi VS. A influência do método Pilates nas atividades de vida diária de idosas [dissertação]. Porto Alegre: Pontifícia Universidade Católica do Rio Grande do Sul; 2009.

10. Freitas EV, Gorzoni LM. Tratado de geriatria e gerontologia. $3^{\mathrm{a}}$ ed. Rio de Janeiro: Guanabara Koogan; 2011.

11. Reis AL, Mascarenhas MHC, Lyra EJ. Avaliação da qualidade de vida em idosos praticantes e não praticantes do método Pilates. CD Fainor. 2011;4(1):38-51.

12. Segura, D. D. C. A., do Nascimento, F. C., Chiossi, C. A., da Silva, M. A. A., Guilherme, J. H., \& Santos, J. V. Estudo comparativo do tratamento da escoliose idiopática adolescente através dos métodos de RPG e Pilates. Saúde e Pesquisa. 2011;4(2):200-2006.

13. Sacco ICN, Andrade MS, Souza PS, Nisiyama M, Cantuária AL, Maeda FYI. Método Pilates em revista: aspectos biomecânicos de movimentos específicos para reestruturação postural-estudos de caso. R. Bras.Ci e Mov. 2005; 13(4):65-78.

14. Ciconelli RM, Ferraz MB, Santos W, Meinão I, Quaresma MR. Tradução para língua portuguesa e validação do questionário genérico de avaliação de qualidade de vida SF-36. Revs Bras Reumatol. 1999;39(3):143-50.

15. Silva LCC. Avaliação funcional pulmonar: incluindo questões de auto-avaliação e respostas comentadas. $2^{\mathrm{a}}$ ed. Rio de Janeiro: Revinter, 2000.

16. Santos M, Cancelliero-Gaiad KM, Arthuri MT. Efeito do método pilates no solo sobre parâmetros respiratórios de indivíduos saudáveis. R. bras. Ci. e Mov 2015;23(1):24-30.

17. Craig C. Pilates com a bola. $2^{\mathrm{a}}$ ed. São Paulo: Phorte 2005.

18. Jesus LT, Baltieri L, Oliveira GL, Angeli RL, Antonio OS, Forti PME. Efeitos do método Pilates sobre a função pulmonar, a mobilidade toracoabdominal e a força muscular respiratória: ensaio clínico não randomizado, placebo-controlado. Fisioter Pesq. 2015;22(3):213-22.

19. Barbosa CWA, Guedes AC, Douglas NB, Silva FA, Martins MLF, Barbosa ASCM . The Pilates breathing technique increases the electromyographic amplitude level of the deep abdominal muscles in untrained people. J Bodyw Mov Ther. 2015;19(1):57-61.

\section{1.}

20. Di Alencar TAM, Matias KFS. Princípios fisiológicos do aquecimento e alongamento muscular na atividade esportiva. Rev Bras Med Esporte. 2010; 16(3):230-34.

21. Santos RR, Silva ALA. Princípios Básicos de Geriatria e Gerontologia, 2. ed. Minas Gerais: Coopmed, 2008.

22. Farinatti VT. Envelhecimento: promoção da saúde e exercício. Bases teóricas e Metodológicas. Barueri: Manole; 2008.

23. Godoy DV, Bringhenti RL, Severa A, Gasperi R, Poli LV. Ioga versus atividade aeróbia: efeitos sobre provas espirométricas e pressão inspiratória máxima. J Bras Pneumol. 2006;32(2):130-35.

24. Gallagher SP, Kryzanowska R. O método de Pilates de Condicionamento Físico. São Paulo: Competition; 2000.

25. Nascimento MM, Carvalho ECF. Comparação da percepção da qualidade de vida entre idosos ativos e iniciantes no método pilates. R. Bras. Qual. Vida. 2015;7(4):259-68.

26. Matsudo SM, Barros NTL. Efeitos benéficos da atividade física na aptidão física e saúde mental durante o processo de envelhecimento. Rev. Bras. Ativ. Fís. Saúde. 2000;5(2):60-76.

27. Chaim J, Raimundo ME, Sant'anna FCA, Yuaso DR. Prática regular de atividade física e sedentarismo: influência na qualidade de vida de idosas. RBCEH. 2010;7(2):198-209.

28. Kolyniak IE, Cavalcanti SMB, Aoki MS. Avaliação isocinética da musculatura envolvida na flexão e extensão do tronco: efeito do método Pilates. Rev Bras Med Esporte. 2004;10(6):487-90. 